\section{Cahiers de Narratologie}

Analyse et théorie narratives

34 | 2018

Les avatars du chapitre en bande dessinée

\title{
Du syntagme au paradigme : les « épisodes » du roman dessiné
}

Jan Baetens

\section{(2) OpenEdition}

\section{Journals}

\section{Electronic version}

URL: http://journals.openedition.org/narratologie/8866

DOI: 10.4000/narratologie.8866

ISSN: 1765-307X

Publisher

LIRCES

\section{Electronic reference}

Jan Baetens, «Du syntagme au paradigme : les « épisodes » du roman dessiné », Cahiers de Narratologie [Online], 34 | 2018, Online since 21 December 2018, connection on 01 May 2019. URL : http://journals.openedition.org/narratologie/8866; DOI : 10.4000/narratologie.8866

This text was automatically generated on 1 May 2019.

Article L.111-1 du Code de la propriété intellectuelle. 


\title{
Du syntagme au paradigme : les " épisodes » du roman dessiné
}

\author{
Jan Baetens
}

\section{Le saut dans le passé : du chapitre à l'épisode}

Inutile de le répéter : le type de bande dessinée « sérieuse » et « exigeante » qu'on appelle aujourd'hui roman graphique (Baetens et Frey 2015) fait un recours fréquent à la technique du chapitrage. Dans son étude Bande dessinée et littérature (2013: $113 \mathrm{sq})$, Jacques Dürenmatt souligne à juste titre que cette technique de composition est un des éléments qui séparent le plus clairement le passage de la bande dessinée traditionnelle à de nouvelles formes de narration graphique par images dessinées ${ }^{1}$. Comme chaque nouveauté, la division en chapitres tend également à créer ses propres précurseurs. Il en va du chapitrage comme de Kafka (dans la célèbre analyse de Borges 2010). C'est en effet à la lumière des procédés contemporains, dûment institutionnalisés, qu'il devient possible de mieux observer les usages antérieurs, mais non encore reconnus comme tels, d'un procédé ou d'un médium (selon un mécanisme qui rappelle celui de la «seconde naissance » étudié par Gaudreault et Marion 2013).

En l'occurrence, l'analyse « littéraire » de la bande dessinée et du roman graphique qui s'est imposée aujourd'hui ne se contente pas de relever l'écart entre l'ancien, défini par le recours à d'autres types de division narrative que le chapitrage, et le moderne, lui fortement marqué par le jeu du chapitre. Outre ce geste de distinction, elle s'efforce également de repenser l'histoire de la division en chapitres, dont certaines formes existaient déjà au moment où les premières lectures scientifiques s'emparaient, pour l'étudier comme pour la défendre, de la bande dessinée, jusque-là activité de pur divertissement (dans le meilleur des cas, car l'innocence du médium était loin de faire l'objet d'un large consensus, voir Beaty 2005, Pizzino 2016 et Groensteen 2017).

Dans les pages qui suivent, on aimerait se pencher sur un ancêtre très particulier de la division en chapitre en bande dessinée, à savoir le recours à l'« épisode », car tel était le terme en usage dans ces années, du roman dessiné, un des multiples avatars de la bande 
dessinée qui annonçaient déjà, du moins selon les points de vue contemporains, ce qui se dégagerait plus tard sous le label de roman graphique. Or, si le roman dessiné illustre la loi borgésienne de l'invention littéraire du passé, il convient de se méfier du "présentisme ", c'est-à-dire de la projection anachronique sur le passé d'idées proprement modernes et contemporaines - démarche presque inévitable (il en va d'ailleurs de même pour toutes nos spéculations sur le futur), mais néanmoins dangereuse, qui empêche de mettre en valeur certaines des leçons les plus intéressantes du passé.

\section{Le roman dessiné, un maillon oublié de l'histoire de la bande dessinée}

Comme l'atteste facilement la lecture de n'importe quelle histoire de la bande dessinée, le roman dessiné est un genre mal connu, voire complètement oublié. Aussi, quelques précisions historiques ne seront pas inutiles pour bien mesurer l'importance de son usage des épisodes à un moment où la notion de chapitre parait encore faire défaut dans le champ plus large de la bande dessinée.

Le roman dessiné a eu sa demi-heure de gloire dans la seconde moitié des années $40 \mathrm{du}$ siècle dernier. Il naît dans l'immédiat après-guerre, même si, techniquement parlant, on continue à en trouver des résidus jusqu'au début des années 60 , voire au-delà ${ }^{2}$. Il s'agit d'une forme de bande dessinée inédite, mélange de mélodrame et de ciné-roman, dont la génération n'a rien de spontané, mais dont l'existence, la forme, le contenu et enfin la politique de diffusion doivent tout au flair de Cino Del Duca, un des nouveaux patrons de la presse, et plus particulièrement de la presse du cœur de cette époque, dont les produits divers de son entreprise, les Éditions mondiales, ont dominé en France des années 1950 aux années 1970 (Antonutti 2013, Serra 2017) ${ }^{3}$.

La forme particulière du roman dessiné est l'aboutissement matériel de deux types de considérations. La première a trait à la notion d'imaginaire. Désireux de lancer de nouveaux produits sur le marché (déjà fort encombré) des magazines féminins, Cino Del Duca, en collaboration avec ses frères restés en Italie, fait le pari de combiner deux formes culturelles qui emportent les suffrages du grand public : la bande dessinée, d'une part, le mélodrame, d'autre part: l'idée de faire des bandes dessinées mélodramatiques visant le lectorat adulte féminin, que l'on retrouvera un peu plus tard aux Etats-Unis avec le lancement des romance comics ${ }^{4}$, est une innovation radicale, à l'impact fulgurant. Ce sera la formule de Grand Hôtel (1946) en Italie, puis de Nous Deux (1947) en France (au début, Nous Deux se limitait à traduire les récits déjà publiés en Italie, mais le succès aidant, la revue pourra développer sa propre stratégie et ses propres créations, voir Giet 1998). Qui plus est, Del Duca tire également profit de l'omniprésence du cinéma, plus particulièrement du cinéma hollywoodien, qui revient en masse sur les écrans européens au moment de la Libération.

La deuxième considération est plus directement économique. Cino Del Duca parvient en effet à élargir considérablement le public des magazines du cœur, produit jusque-là typiquement middle-brow, en divisant par trois le prix de son nouvel hebdomadaire (le premier numéro de Grand Hôtel se vendait à 12 lire et celui de Nous Deux à 10 francs, soit respectivement 0,39 euros en 2014 et 0,55 euros en $2016^{5}$ ). Ce faisant, il touche un public 
moins lettré, qui achète pour la première fois un imprimé périodique, mais où l'essentiel passe par l'image et non plus par le texte (même si le texte en est tout sauf absent).

Mélangeant économie et imaginaire, la proposition de Cino Del Duca consiste donc à introduire dans la presse du cœur une nouvelle forme susceptible de répondre à toutes ces contraintes aussi bien qu'à tous ces besoins. Grand Hôtel, le magazine du roman dessiné, puis Nous Deux, le magazine du bonheur, obtiennent dès leur lancement un succès phénoménal, qui en fait tout de suite le modèle de tous les autres magazines sur le marché. La clé de la réussite est sans conteste le roman dessiné, puis le roman-photo, inventé en 1947 par les concurrents de Del Duca, mais que celui-ci réussira, il est vrai non sans retard, à intégrer à ses propres publications.

Mais qu'est-ce que le roman dessiné ? C'est une forme de bande dessinée qui :

- reprend l'univers du mélodrame (dont le thème majeur, qu'on retrouve également chez Hitchcock, du moins dans les premières analyses des Cahiers du cinéma, est celui de l'innocence persécutée et dont l'omniprésence culturelle dans les années d'après-guerre est soulignée par plus d'un critique, voir Morreale 2011) ;

- adopte une forme visuelle, à savoir le dessin au lavis exécuté dans l'esprit du réalisme photographique, qui brouille les frontières entre dessin et cinéma ;

- plagie l'iconographie du cinéma populaire, américain aussi bien que local, notamment quand il s'inspire des vedettes de l'image pour façonner le corps et le visage des protagonistes de ses histoires, sans couper les ponts avec les traditions écrites du mélo et de la romance ; le roman dessiné feint copier les scénarios "vus à l'écran ", mais n'oublie pas de les ancrer dans les stéréotypes de la littérature populaire héritée du XIX ${ }^{\mathrm{e}}$ siècle ;

- s'adresse exclusivement à un lectorat féminin (quand bien même toutes les enquêtes et de nombreux témoignages attestent l'existence d'un important lectorat masculin);

- embrasse de manière exacerbée la logique narrative du feuilleton, chaque livraison juxtaposant les épisodes de plusieurs histoires dessinées en cours ;

- s'identifie fortement au format du magazine, et vice versa, les autres rubriques tendant à s'effacer petit à petit au profit du seul roman dessiné ; cette identification est due aussi à des raisons économiques: pour le public populaire visé par Del Duca, l'abonnement n'est pas une option, parce que dépassant la limite du budget personnel, d'où la nécessité de maintenir sans arrêt le désir d'achat au numéro.

Et il convient d'ajouter un point capital pour la présente analyse : le roman dessiné est une bande dessinée qui se construit à partir de la notion d'épisode, généralement entre quatre et six pages hebdomadaires. La notion de chapitre, l'épisode étant clairement composé comme un chapitre, est d'autant plus décisive que la publication en revue était souvent la seule possible, la reprise du récit complet ou la production de récits inédits n'étant pas la norme (on verra plus loin ce qui arrive lorsque des magazines de roman dessiné comment à republier les histoires les plus populaires sous forme de récits complets, voire à lancer des collections de romans dessinés complets inédits - pratique relativement rare, mais non inexistante). Dit autrement : les auteurs d'un roman dessiné ne travaillaient pas en vue d'une réédition sous forme de numéro spécial ou de la reliure de plusieurs numéros, contrairement à ce qui deviendra la norme au tournant éditorial représenté par la généralisation de l'album comme support privilégié de publication (Revaz et Boillat 2013). Ces rééditions et ces publications indépendantes existent, mais la formule de base est sans conteste celle du feuilleton " pur ». 


\section{Métamorphoses de l'épisode dans les magazines de roman dessiné}

Si l'on accepte que la logique narrative de l'épisode («puntata » en italien) est celle du feuilleton, que peut-on apprendre de telle parenté sur la construction du chapitre en roman dessiné ? Logiquement, on devrait retrouver dans chaque épisode la structure ternaire de l'unité feuilletonesque : un début, un milieu, une fin, soit plus concrètement : d'abord le rappel, même très succinct, des événements passés ; ensuite une nouvelle étape dans le déroulement de l'action; enfin la production d'un effet d'attente, idéalement sous forme de cliffhanger puisque la survie économique des magazines du roman dessiné dépend de l'achat au numéro. Dans les épisodes du roman dessiné, cette structure ternaire est indéniablement présente, mais elle est actualisée d'une manière plus complexe qu'on ne le pense de prime abord. Trois éléments doivent retenir ici l'attention, dont la convergence signale un écart certain par rapport au modèle classique des épisodes d'un feuilleton.

Premièrement, la composition ternaire est réagencée par l'expansion et la complexification du milieu. Dans les formes les plus élaborées du genre (pour une présentation détaillée des intrigues, qui prend à chaque fois plusieurs pages, voir Cardone 2004), on constate certes que le roman dessiné maintient et exploite les fonctions de relance initiale et de tension finale, mais le trait le plus voyant de chaque épisode est sans conteste la prolifération et l'excès narratifs dans le segment du milieu. Au lieu de construire l'épisode autour d'une nouvelle action ou d'une seule péripétie, le roman dessiné se plaît souvent à multiplier les fils narratifs et à poursuivre le récit en plusieurs lieux et en plusieurs couches temporelles, à mettre en avant plusieurs voix narratives, enfin à mélanger plusieurs intrigues provisoirement indépendantes. L'effet qui en résulte est paradoxal. D'une part, l'épisode remplit parfaitement sa fonction dans la logique générale du feuilleton. D'autre part, chaque épisode tend à se transformer en microrécit, voire en récit complet lui-même, cessant dès lors d'être seulement un des maillons de la chaîne du feuilleton et devenant une histoire sinon autosuffisante (commercialement, une telle stratégie serait suicidaire), du moins suffisamment étoffée pour donner une impression de complétude (qui rassure l'acheteur quant au rapport qualité/prix du magazine). Il se passe en d'autres mots tant de choses entre le début et la fin, que le milieu peut se lire comme un récit presque indépendant - à quoi il importe d'ajouter que les pages des magazines étaient souvent très grandes et que contrairement à un préjugé tenace le texte y occupait parfois autant sinon davantage de place que les images proprement dites. Cette métamorphose ne reste pas sans effet sur le début et la fin des épisodes, qui du coup perdent quelque peu en importance, le milieu de chaque épisode étant lui-même le lieu de plusieurs microséquences ayant chacune leur début (leur rappel), leur milieu (leur action centrale) et leur fin (leur suspense).

En second lieu, l'épisode n'est jamais seul de son genre. En principe, les magazines du roman dessiné ne publient pas un seul feuilleton, ils se présentent comme une mosaïque de feuilletons, qui se suivent tout en alternant avec quelques autres rubriques à vocation également sérielle, tels les lettres à l'éditeur, l'horoscope ou les cancans mondains (la mode et la cuisine ne sont pas encore à l'honneur en ces années où le rationnement est toujours de rigueur). Cette construction en polyptyque feuilletonesque porte à conséquence pour la lecture des épisodes, lus d'abord l'un après l'autre, puis l'un à la 
lumière et, plus encore, en fonction de l'autre, comme s'ils constituaient les chapitres successifs d'un grand récit implicite raconté entre les deux couvertures d'un numéro de magazine. À première vue, cette hypothèse paraît gratuite si ce n'est absurde, mais il suffit de se rendre compte de l'incroyable unité thématique et générique des divers récits en cours, qui sont tous des mélodrames et qui mettent sans exception l'accent sur les questions du couple, souvent sous la forme de personnages historiques bien connus des amateurs d'une certaine littérature populaire (la reine Marie-Antoinette ou l'impératrice Sissi, par exemple, ou encore Anna Karénine ou Madame Bovary - en version reader's digest bien entendu), pour que l'idée d'une continuation narrative au-delà des intrigues différentes acquière une certaine plausibilité. D'autres facteurs soutiennent cette interprétation : la relative autonomie de chaque épisode, par exemple, qui fonctionne à la fois comme fragment (du feuilleton) et comme tout (c'est-à-dire comme récit complet), mais aussi la familiarité certaines du public avec les règles et les stéréotypes du mélodrame, sur lesquelles le roman dessiné revient inlassablement (chaque récit est différent, mais sa différence tient à la réarticulation des motifs et des noyaux narratifs bien connus des lecteurs). Bref, le lecteur ou la lectrice de ce type de magazines est un lecteur ou une lectrice qui s'intéresse non seulement au contenu des récits, mais aussi à la manière de les raconter, c'est-à-dire à la tentative de répéter de manière légèrement différente des matières qu'on connaît pourtant par cœur. Typique de n'importe quelle industrie culturelle, pareil parti pris facilite l'appréciation des épisodes " hors récit », à la fois en eux-mêmes et dans le contexte matériel qui est le leur. On ne lit pas seulement tel ou tel épisode dans l'espoir de connaitre la fin de l'histoire, mais aussi en le comparant à ce qui le suit ou le précède dans tel ou tel numéro du magazine. Telle autonomisation de l'épisode, qui se détache du récit complet dont il fait partie pour contracter de nouvelles unions avec les épisodes et fragments alentour, est suggérée par les caprices ou nonchalances des reliures proposant l'achat groupé de plusieurs livraisons, qui ne font pas nécessairement suite. Ce qu'on vend comme reliure, ce sont littéralement des numéros du même magazine, mais dont la série est souvent trouée (c'est le cas des reliures 4 Tendres Idylles, qui reprennent des numéros du magazine Idylles écran, mais dans un ordre discontinu').

Une troisième particularité des épisodes dans le roman dessiné tient à un infléchissement des effets d'annonce. Loin de se réduire à une technique interne au roman - la création d'un effet de suspense en fin de chapitre (Baroni 2017) - la fonction d'appel essaime dans tout le magazine. La publication de tel ou tel épisode ne sert pas uniquement à pousser à l'achat du numéro suivant, elle fournit surtout l'occasion de multiplier à plusieurs endroits et de plusieurs manières l'incitation à ne pas laisser passer le numéro en question - et il importe de garder à l'esprit que les numéros n'étaient disponibles en kiosque que pendant une semaine ou un mois seulement, suivant la périodicité de la publication -, mais aussi à se procurer d'autres revues ou magazines du même éditeur, le catalogue de chaque maison misant toujours sur la multiplication de séries de prime abord très différentes mais en réalité parfaitement similaires. En bas de nombreuses pages comme dans ces vignettes publicitaires publiées en médaillon, on annonce aussi bien la suite du feuilleton que le début de nouvelles histoires, tous détails utiles à l'appui. Le mécanisme est le même quel que ce soit le type de publication. Dans Accroche-cœur (éditions Imperia de Lyon), qui ne publie qu'un feuilleton à la fois, ces indications péritextuelles fournissent toujours le même type d'informations: date de parution, nombre de pages, prix (élément essentiel de n'importe quel produit des industries culturelles), adresse du magazine, caractère « inédit » du roman dessiné, invitation à se 
procurer les numéros déjà parus auprès de l'éditeur - mais pas d'invitation à l'abonnement. Dans OUI (éditions des Remparts, également basées à Lyon), qui choisit la formule nettement moins fréquente du récit complet, un nombre considérable de pages est consacré à l'annonce de nouveaux récits, soit par le biais d'inserts publicitaires, soit à travers de véritables prépublications (dont les pages sont reprises telles quelles dans le numéro suivant), un peu à la manière des trailers au cinéma. Dans Idylle écran (« éditée par la société d'imprimerie et d'édition du Puits-Pelu, Jacquier et Cie », toujours à Lyon), qui mélange nouvelles complètes et feuilletons, les récits se terminent souvent par une grande case annonçant le contenu des numéros à venir.

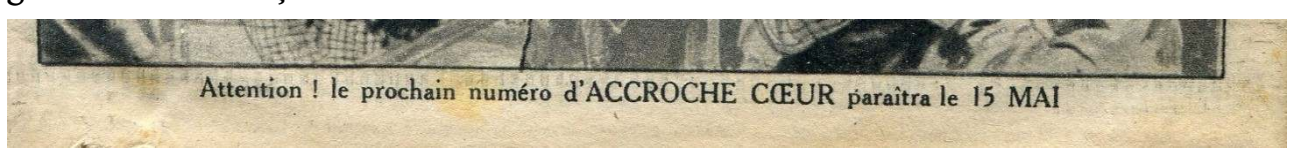

Figure 1a/b/c : Inserts publicitaires en bas de page dans Accroche-cœur. 6 numéros complets, Lyon, éd. Impéria, 1949)

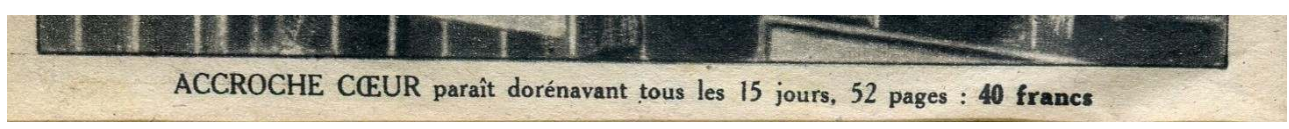

Figure 1a/b/c : Inserts publicitaires en bas de page dans Accroche-cœur. 6 numéros complets, Lyon, éd. Impéria, 1949)

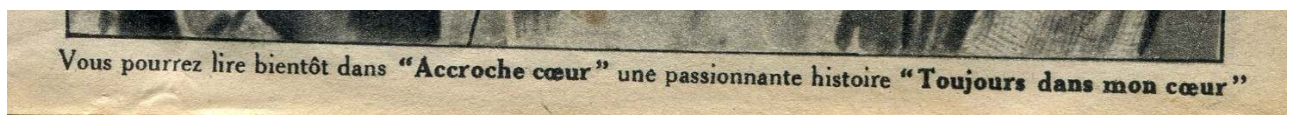

Figure 1a/b/c : Inserts publicitaires en bas de page dans Accroche-cœur. 6 numéros complets, Lyon, éd. Impéria, 1949)

\section{Du feuilleton au récit complet : du même au même?}

Rien ne change lorsque le genre du roman dessiné quitte le domaine du feuilleton pour aller résolument vers la publication de récits complets, sans doute pour se distinguer du roman-photo qui avait commencé à prendre sa place dans les magazines du cœur et qui renchérissait encore davantage sur le modèle du feuilleton, avec des effets d'attente et de relance souvent sensationnels - probablement à cause de la structure plus simple, narrativement parlant, du nouveau médium. Un des traits les plus frappants des épisodes du roman-photo est la simplification de ce qu'on a appelé plus haut le «milieu» de l'épisode. Dans le roman-photo, qui raconte « moins » que le roman dessiné - moins de personnages, moins de péripéties, moins de lieux, moins de strates temporelles -, mais qui met tout en œuvre pour faire valoir son atout majeur, la présence quasi-physique, tactile, littéralement touchable, du corps des acteurs, il y a tout simplement moins de place pour étoffer la partie centrale de chaque épisode. La concurrence initiale entre magazines de roman dessiné (Grand Hôtel et Nous Deux, avant qu'ils ne se convertissent aux récits photographiques) et magazines de roman-photo (essentiellement Il Mio Sogno et Bolero-Film, très lus aussi dans la diaspora italienne) fera le reste: le roman dessiné s'efforcera logiquement de monter en épingle un élément qui lui permet de se distinguer du roman-photo, à savoir la publication de récits complets aussi longs que le sont parfois les livraisons accumulées du roman-photo (souvent de douze à quinze épisodes de quatre ou six pages - la longueur des romans dessinés complets est visiblement calqué sur celle des épisodes mis bout à bout du roman-photo). 
Mais donnons d'abord quelques exemples de la nouvelle pratique éditoriale: Nous Deux, leader du marché de la presse du cœur mais tard venu dans le monde du roman-photo qu'il choisit de garder à l'écart pendant plusieurs années (en fait jusqu'à ce que les lois de la concurrence l'obligent à inclure le nouveau médium moins cher à produire et jugé plus moderne par un public affamé de nouveautés, Voir Minuit, Faber et Takodjerad 2012), lance une série de romans dessinés complets et inédits, non pas à l'intérieur du magazine, mais en marge, dans une série spécialisée, pratique les mêmes effets publicitaires, qui ne sont plus des effets d'annonce ("à suivre »), mais de véritables effets de catalogue («du même éditeur »), toutes collections confondues. La pratique est générale dans l'édition populaire : les pages disponibles des «Grands Romans "noirs" dessinés » (une publication des Presses Mondiales, à ne pas confondre avec les Éditions mondiales) ou de la «Collection Frissons » (éditions de la Flamme d'Or, Paris, qui est aussi la société derrière les éditions du Rempart) servent ainsi à faire la promotion d'autres collections - et vice versa.

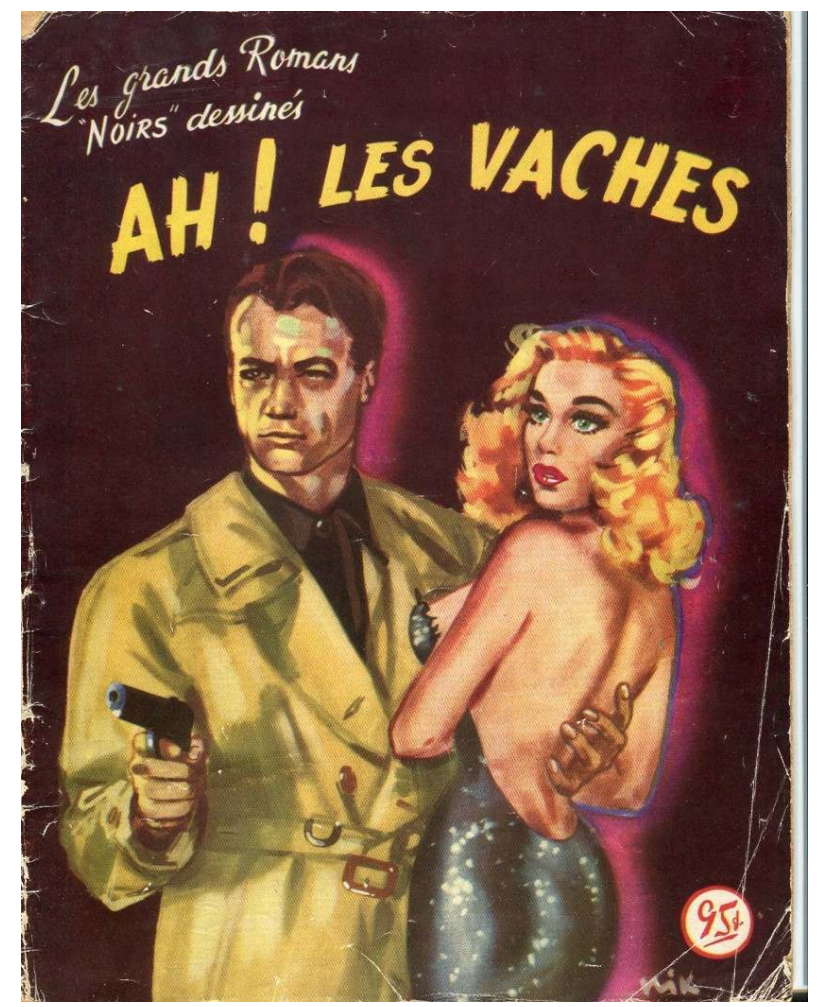

Figure 2a/b : Première et quatrième de couverture de Ah ! les vaches, Paris, Presses Mondiales, n.d., coll. "Les grands Romans "noirs" dessinés » 


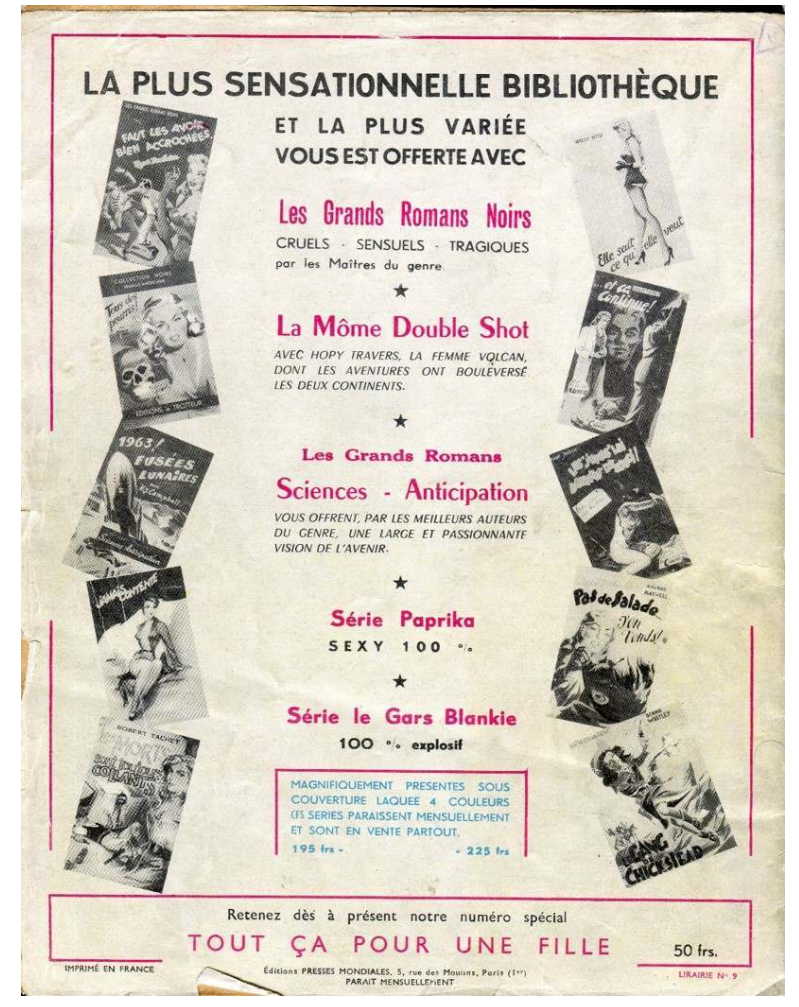

Figure 2a/b : Première et quatrième de couverture de Ah ! les vaches, Paris, Presses Mondiales, n.d., coll. « Les grands Romans "noirs" dessinés »

Ici encore, la fonction publicitaire semble se détacher de l'effet d'annonce, et ce doublement : d'abord, et assez logiquement, par l'abandon du feuilleton (disparation de l'effet «à suivre »), ensuite, et de manière plus intéressante, par un déplacement de l'objet de l'appel publicitaire (ce qu'on cherche à vendre, ce n'est plus «la suite au prochain numéro ", mais d'autres titres de la collection, voire d'autres séries de la même maison d'édition).

Une conclusion provisoire s'impose : tout semble se passer comme si l'épisode du roman cessait de fonctionner dans la perspective du syntagme (l'épisode comme maillon d'une chaîne allant du début à la fin du récit) pour s'inscrire dans une tout autre logique, celle du paradigme (en l'occurrence l'ensemble des publications de la presse du cœur, l'épisode devenant un élément de la classe des magazines de femmes, susceptible de servir de pivot ou de carrefour vers n'importe quelle autre unité du paradigme).

\section{Des chapitres qui n'en sont pas}

Cette analyse exhibe utilement ce qui persiste d'un mode de publication à l'autre, mais dit peu de ce qui change. Or, les transformations ne sont pas moins notables que les continuations - en l'occurrence celles de la double structure narrative et publicitaire des œuvres -, et l'une d'elles touche directement à la gestion des chapitres. En effet, lorsque le roman dessiné évolue du mode du feuilleton au régime du récit complet, cette transition a un impact brutal sur le chapitrage, qui disparait en tant que principe d'organisation narrative. Le récit se fait à la fois plus continu (on ne retrouve plus les segments du feuilleton, la narration devient plus fluide) et moins complexe, moins baroque (on perd également la nécessité de transformer chaque maillon de la chaîne en 
microrécit complet, ce qui se perd en termes de densité se compense en termes de longueur et à ce niveau il est essentiel de ne pas trop solliciter la capacité d'attention du lecteur). Techniquement parlant, la composition en chapitres aurait pu se maintenir sans problème. Culturellement parlant toutefois, le chapitre "connotant» la littérature légitime, elle devient une hypothèse non seulement inutile mais aussi nocive. Un accent trop fort mis sur le chapitrage aurait risqué de pousser le récit vers une sophistication dont le genre du roman dessiné avait besoin dans sa version feuilletonesque, mais qui aurait nui au régime de consommation rapide et légère qui était le sien en «version complète ».

Il est cependant des cas où la charpente du chapitrage survit à l'abandon du feuilleton. Amour dans l'ombre (collection "Nous Deux présente", 1953) en offre un exemple passionnant. À intervalles réguliers, en moyenne toutes les sept pages, le récit prévoit une pause, qui adopte fidèlement la forme d'un paratexte d'épisode: une nouvelle séquence paraît s'ouvrir, avec en haut de page une image panoramique, toujours la même du reste, une vue d'ensemble de la ligne des toits de Montmartre, qui semble donner un récapitulatif de l'épisode écoulé. "Paraît », "semble»: le choix des mots révèle déjà à quel point le mécanisme du chapitrage reste superficiel et anecdotique : non seulement le compartimentage en unités de longueur plus ou moins comparable répond peu à la structure du récit proprement dit, qui aurait pu s'interrompre à bien d'autres endroits, mais le résumé qu'on trouve dans les frises au seuil des épisodes, d'ailleurs sans numéro ni titre, ne diffère pas en rien de ce qu'on trouve en bien d'autres cases explicatives qui ponctuent et complètent le récit visuel. En d'autres mots, la division en épisodes est un simulacre de chapitrage, non une transposition de la notion de chapitre du feuilleton au récit complet.

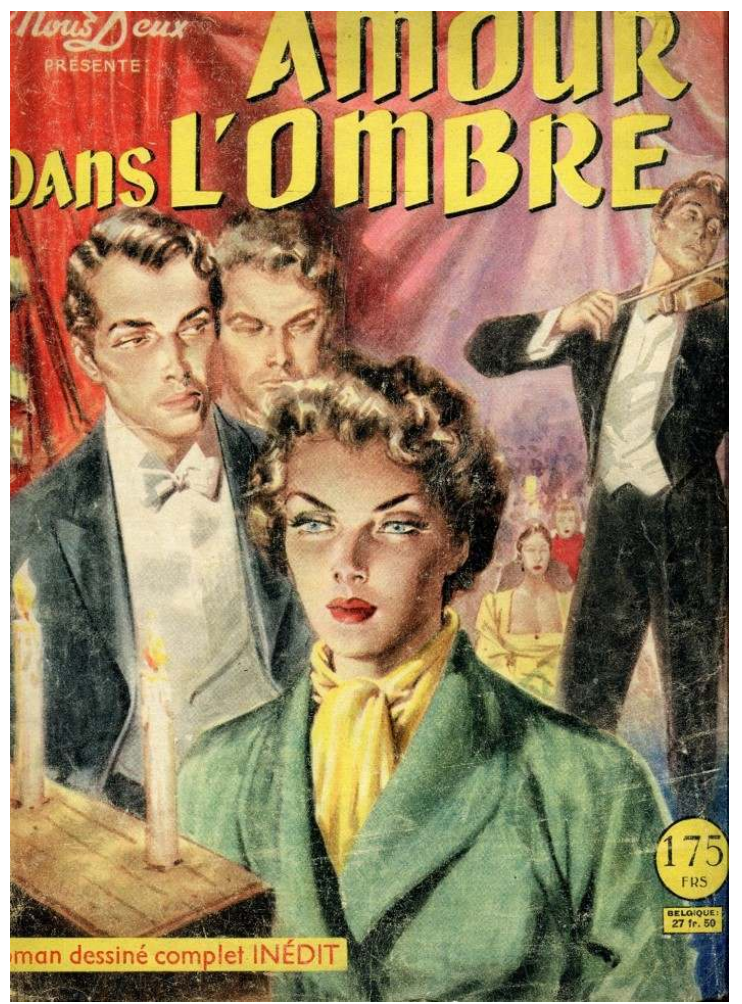

Figure 3a : Première de couverture du récit complet Amour dans l'ombre, Paris, Éditions Mondiales, coll., « Nous Deux présente », 1953. 


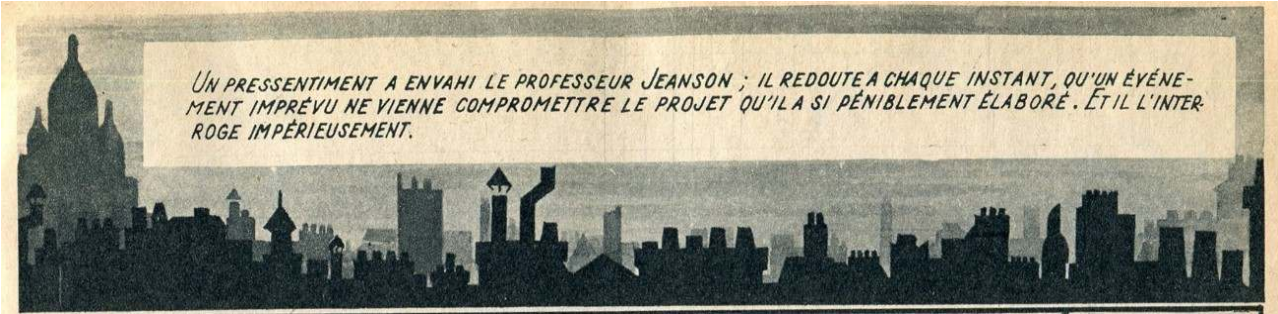

Figures $3 \mathrm{~b} / \mathrm{c} / \mathrm{d}$ : Récapitulatifs insérés en haut ce certaines pages (non paginées) d'Amour dans l'ombre

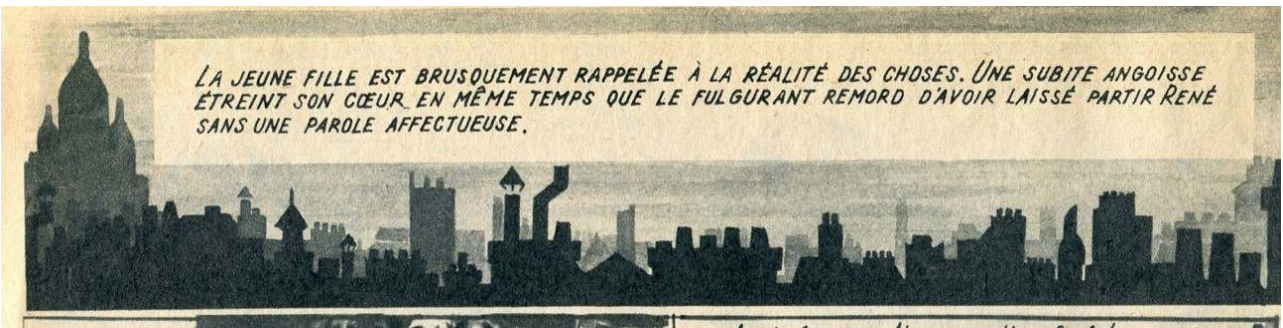

Figures $3 b / c / d$ : Récapitulatifs insérés en haut ce certaines pages (non paginées) d'Amour dans l'ombre

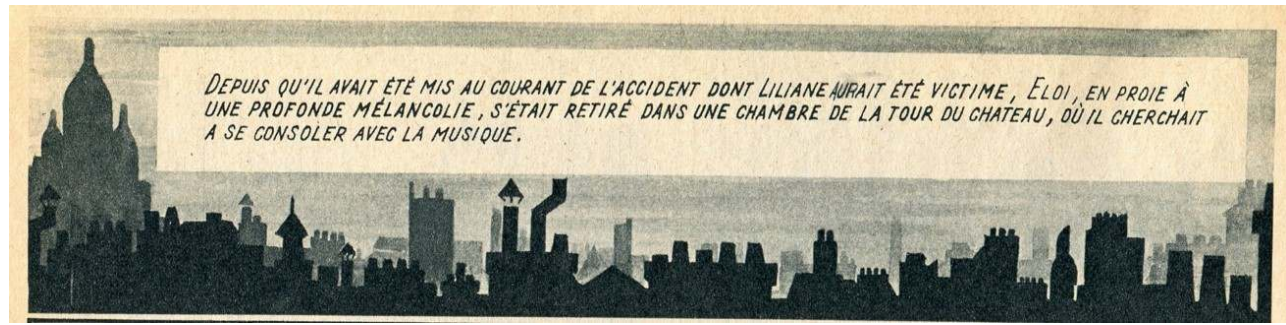

Figures 3b/c/d : Récapitulatifs insérés en haut ce certaines pages (non paginées) d'Amour dans l'ombre

Cela dit, simulacre ne signifie pas absence de fonction ou de sens. En l'occurrence, la signification du pseudo-chapitrage est profondément liée au changement de sens de l'épisode, dont l'horizon d'attente n'est plus seulement le syntagme mais aussi le paradigme. Pour le roman dessiné de type "post-feuilleton », il est en effet crucial de garder un lien direct avec le monde des magazines, parce qu'il faut éviter d'aliéner le public populaire, plus proche des feuilletons que des récits complets, qui tirent la littérature populaire vers l'imaginaire du livre typique de la littérature légitime. En simulant le chapitrage au sein d'une forme de récit qui aurait pu s'en passer, on essaie de rassurer le lecteur, d'abord en lui rappelant les origines feuilletonesques du genre, ensuite en lui promettant les qualités, par exemple de suspense et de grand rythme, qui font le prix du feuilleton aux yeux du lectorat populaire. Le découpage narratif en épisodes semble survivre "pour rien", comme un écho anachronique et un peu paresseux des premiers temps du genre, comme si les auteurs du scénario n'avaient pas encore désappris l'usage du chapitre. En réalité, toutefois, le maintien des épisodes, si privé de fonction narrative soit-il, correspond à de profonds besoins culturels. Il signale aux lecteurs que les ponts avec le monde des magazines ne sont pas coupés et que l'achat d'un récit complet, tout en offrant un ersatz de standing et de prestige (on n'est pas encore dans le monde du livre, mais on n'est plus non plus dans celui des magazines traditionnels), n'est pas incompatible avec les plaisirs et l'excitation garantis par les revues de romans dessinés ou de romans-photos. 
Si la division en chapitre est un mode de composition qui peut s'appliquer à n'importe quel récit, il serait erroné d'en conclure que l'universalité de la forme implique aussi une identité des usages et des fonctions. Cette diversité doit s'étudier d'abord au plan narratif et stylistique, mais on ne peut en exclure les aspects culturels, intimement liés à des contextes de création, distribution et réception extrêmement précis. L'exemple des emplois de l'épisode dans le roman dessiné, un genre habituellement absent des discussions sur les structures du récit, montre ainsi que le chapitre signifie à la fois par présence et par absence et que les enjeux de ses apparitions ou disparitions ne peuvent être saisis que dans une perspective historique, culturelle et intertextuelle. Le chapitrage d'un roman dessiné ne sert pas toujours un but narratif ou stylistique et dans certains cas il se présente en trompe-l'œil, sous forme de simulacre. On peut gager cependant que ces particularités ne sont pas propres au seul roman dessiné, mais qu'elles aideront à poser de nouvelles questions dans l'analyse de genres mieux balisés, culturellement plus légitimes.

\section{BIBLIOGRAPHY}

Antonutti, Isabelle (2013), Cino Del Duca, de Tarzan à Nous Deux, Limoges, PULIM.

Baetens, Jan \& Hugo Frey (2015), The Graphic Novel. An Introduction, New York, Cambridge University Press.

Baroni, Raphaël (2017), Les Rouages de l'intrigue, Genève, Slatkine.

Beaty, Bart (2005), Fredric Wertham and the Critique of Mass Culture, Jackson, The University Press of Mississippi.

Borges, Jorge Luis (2010), « Kafka et ses précurseurs », CEuvres complètes I, Paris, Gallimard, Bibliothèque de la Pléiade, dir. Jean-Pierre Bernès, p. 751-753.

Cardone, Lucia (2004), Con lo schermo nel cuore. Grand Hôtel e il cinema (1946-1956), Pisa, Edizioni ETS.

Dürrenmatt, Jacques (2013), Bande dessinée et littérature, Paris, Garnier.

Frey, Hugo \& Jan Baetens (2018), « Comics Culture and Roy Lichtenstein Revisited : Analyzing a Forgotten "Feedback Loop" ", Art History (à paraître).

Gaudreault, André \& Philippe Marion (2013), La Fin du cinéma ?, Paris, Colin.

Giet, Sylvette (1998), Nous Deux 1947-1997. Apprendre la langue du cœur, Louvain \& Paris, Peeters \& Vrin.

Groensteen, Thierry (2017), La Bande dessinée au tournant, Bruxelles, Les Impressions Nouvelles.

Minuit, Marion, Dominique Faber \& Bruno Takodherad (2012), Nous Deux présente la saga du romanphoto, Paris, Gawsewitch.

Morreale, Emiliano (2011), Così piangevano. Il cinema melò nell'Italia degli anni Cinquanta, Rome, Donzeli. 
Pizzino, Christopher (2016), Arresting Development. Comics at the Boundaries of Literature, Austin, Texas University Press.

Potet, Frédéric (2017), « Sentimental, Fabcaro ? », Le Monde des livres, 22 décembre, p. 9.

Revaz, Françoise \& Alain Boillat (dir.) (2013), La BD avant l'abum, Lausanne, GReBD (catalogue d'une exposition au Forum de l'Hôtel de Ville de Lausanne du 16 au 26 décembre 2013).

Serra, Hubert (2017), Voyage au cœur du roman-photo. Autobiographie, Paris, Les Indes savantes.

\section{NOTES}

1. Voir également, ici-même, Raphaël Baroni, « Le chapitrage dans le roman graphique américain et la bande dessinée européenne : une segmentation précaire ».

2. On en veut pour exemple le compte rendu du dernier album de Fabcaro, Et si l'amour c'était aimer? (Montpellier, 6 pieds sous terre, 2017) : «Ceux qui n'auront pas l'occasion de visiter l'exposition "Roman-photo" au MuCEM, à Marseille, se consoleront amplement en achetant le nouvel album de Fabcaro, figure de proue de la BD indépendante et dynamiteur attiré des modes de vies contemporains. L'auteur (...) s'est emparé des codes de la romance sur papier glacé en réalisant un roman-photo... sans photo, entièrement dessiné à partir de magazines de types Nous deux.» (Potet $2017: 9$ ).

3. Afin de motiver le mot de «flair », voici une anecdote racontée par Hubert Serra : « En matière d'édition, rien ne vaut le flair. L'assurance que ce l'on fait correspond bien au public que l'on veut toucher. Les études de marchés sont intéressantes mais à titre d'information seulement. Au temps où j'étais metteur en pages chez Del Duca, il y avait une jeune femme, choyée comme une princesse par le patron, dont la fonction consistait à lire nouvelles et romans. Si elle pleurait ou était émue par un texte, il était approuvé aussitôt pour l'édition. Del Duca faisait grand cas du sens particulier de cette femme pour l'émotion, car il devait penser que son public était de même nature » (Serra $2017: 138$ ).

4. Le genre sera lancé en 1947 par le magazine "Young Romance », dirigé par Jack Kirby et Joe Simon. Aujourd'hui, on s'en souvient le mieux à travers les appropriations "pop » de Roy Lichtenstein, mais ce rapprochement un peu facile ne rend pas justice à la complexité des rapports entre bande dessinée et arts plastiques de ces années (Frey et Baetens 2018).

5. Pour la conversion des lires en euros, voir le convertisseur du journal Il Sole: http:// www.infodata.ilsole24ore.com/2015/04/14/se-potessi-avere-calcola-il-potere-dacquisto-in-lireed-euro-con-la-macchina-del-tempo/ (consulté le 28 décembre 2017).

Pour la conversion des anciens francs en euros, voir le convertisseur de l'INSEE: https:// www.insee.fr/fr/information/2417794 (consulté le 28 décembre 2017).

6. Toutes proportions gardées, on pourrait risquer la même déduction en examinant les lots offerts sur eBay, qui sont presque toujours des ensembles tout à fait dépareillés. Ce manque de cohésion interne désigne peut-être le caractère imprévisible des achats: peu portés sur l'abonnement, trop coûteux, les lecteurs semblaient intéressés davantage par le "genre ", quelle que soit la publication concernée, que par le souci d'avoir la série complète de tel ou tel magazine. 


\section{ABSTRACTS}

The purpose of this study is twofold. First, to analyze the place and function of the chapter in a little-known genre, the "roman dessiné ». This analysis is based on a comparative approach to this almost forgotten cultural practice, at the crossroads of comics, cinema and photo-novel. Secondly, to uncover some functional features of the chaptering of the novel drawn in relation to its modes and media of publication (serial narrative versus full narrative, circulation within women's magazines), in the hope of raising new questions in the study of the chapter in general.

La présente étude se donne un double but. Premièrement, analyser les place et fonction du chapitre dans un genre peu connu, le roman dessiné. Cette analyse s'appuie sur une approche comparée de cette pratique culturelle quasiment oubliée, au carrefour de la bande dessinée, du cinéma et du roman-photo. Deuxièmement, mettre au jour certaines particularités fonctionnelles du chapitrage du roman dessiné en rapport avec ses modes et supports de publication (récit en feuilleton versus récit complet, circulation à l'intérieur de magazines féminins), dans l'espoir de faire surgir de nouvelles interrogations dans l'étude du chapitre en général.

\section{INDEX}

Mots-clés: chapitre, roman dessiné, magazine féminin

Keywords: chapter, roman dessiné, women's magazine

\section{AUTHOR}

\section{JAN BAETENS}

Jan Baetens est professeur d'études littéraires et culturelles à l'université de Leuven. Il est l'auteur de plusieurs ouvrages sur la poésie, dont À voix haute. Poésie et lecture publique (Les Impressions Nouvelles, 2016), et sur les rapports entre texte et image, dont Pour le roman-photo (Les Impressions Nouvelles, 2017), ainsi que d'un roman (Faire sécession, L'herbe qui tremble, 2017) et de plusieurs recueils de poésie, dont Vivre sa vie et autres poèmes (Espace Nord, 2014). Courriel : jan.baetens@kuleuven.be 